\title{
A CMOS Low Dropout Regulator Stable With Any Load Capacitor
}

\author{
Hsuan-I Pan, Chin-Hung Cheng, Chern-Lin Chen, Senior Member IEEE \\ Department of Electrical Engineering \& \\ Graduate Institute of Electronics Engineering \\ National Taiwan University
}

Ta-yung Yang

System General Corp. Taiwan

\begin{abstract}
A CMOS low dropout regulator (LDO) with a new pole-zero pairs cancellation compensation scheme is presented. LDO using this proposed compensation technique provides stable output voltage with any load capacitor. The only constraint on the equivalent series resistance (ESR) of the load capacitor is that it should be less than the load resistance. It is especially suitable for system-on-chip integration applications and board size reduction.
\end{abstract}

\section{INTRODUCTION}

Low dropout regulators (LDOs) are widely used for power management applications. Compared with switching regulators, LDOs are less expensive, smaller in size and easier to be used. Moreover, the noise of output voltage is lower and the response to input voltage transient and output load transient is faster. These advantages make LDOs suitable for battery-powered equipments, communication systems, portable systems, and post regulators of switching regulators. Among possible process technologies, CMOS technology is very attractive for LDO circuit implementation because of its low cost, low power consumption and potential for future system-on-chip integration.

One of the most important issues in LDO design is to maintain the system stability. Conventionally, all LDO systems require off-chip load capacitors for stability. In addition to the value of the load capacitor, the capacitor's parasitic equivalent series resistance (ESR) plays an important role [1]. Most LDOs rely heavily on the ESR value for stability. The basic problem with such LDOs is that the ESR, being parasitic, is not well controlled and not guaranteed by capacitor manufacturers, specifically at low temperatures.

In the literatures, there have been several LDO compensation methods [2 6] presented. These methods mostly focus on improving the performance of LDO. The ESR and the value of the load capacitor are still limited. A damping-factor-control frequency compensation approach [7] is presented to allow a capacitor-free CMOS LDO.

In this paper, a CMOS LDO using new compensation scheme that can maintain system stability without a load capacitor is proposed. Moreover, it can provide stable output under all load conditions with any value of load capacitor. The ESR of the load capacitor can range from zero to some finite value.

\section{REVIEW OF CONVENTIONAL LDO COMPENSATION SCHEME}

Fig. 1 shows a conventional LDO system. PMOS is used as the pass element to improve dropout voltage performance without increasing complexity of the circuit [8]. A conventional LDO can be considered a two-stage amplifier connected as a unity gain buffer. The first stage is the error amplifier, and the second stage consists of the pass element with $\mathrm{R}_{1}, \mathrm{R}_{2}, \mathrm{R}_{\mathrm{L}}$, $\mathrm{R}_{\mathrm{ESR}}$ and $\mathrm{C}_{\mathrm{L}}$. Due to high output resistance of the error amplifier and large $\mathrm{C}_{\mathrm{L}}$, there is one low frequency pole at the output of each stage, respectively. Conventionally, the $\mathrm{R}_{\mathrm{ESR}}$ is utilized to insert a zero between two low frequency poles and unity gain frequency, as shown in Fig. 2. Normally, $\mathrm{R}_{1}, \mathrm{R}_{2}$ are much larger than $\mathrm{R}_{\mathrm{L}}$.

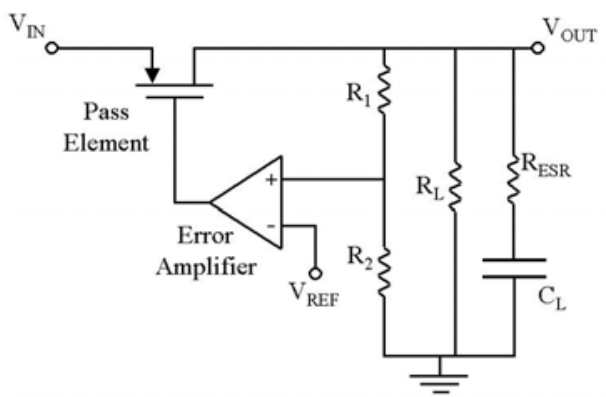

Fig. 1 System block of a conventional LDO 


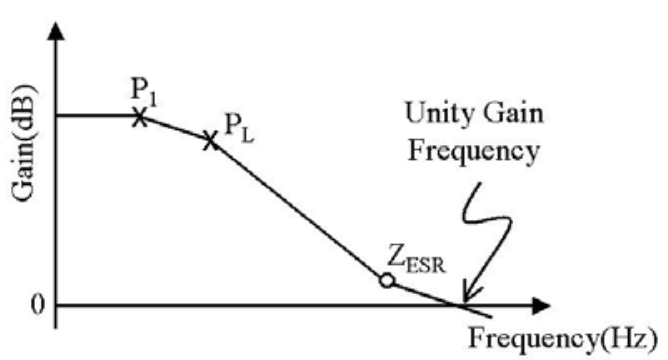

Fig. 2 Frequency response of the LDO open loop gain with conventional compensation scheme

The low frequency poles and ESR zero in open loop transfer function can be estimated as

$$
\begin{aligned}
& \mathrm{P}_{1}=\left(2 \pi \mathrm{C}_{\mathrm{PAR}} \mathrm{R}_{\mathrm{OA}}\right)^{-1} \\
& \mathrm{P}_{\mathrm{L}}=\left(2 \pi \mathrm{C}_{\mathrm{L}} \mathrm{R}_{\mathrm{L}}\right)^{-1} \\
& \mathrm{Z}_{\mathrm{ESR}}=\left(2 \pi \mathrm{C}_{\mathrm{L}} \mathrm{R}_{\mathrm{ESR}}\right)^{-1}
\end{aligned}
$$

$P_{1}$ is the pole at output of the error amplifier stage and $\mathrm{P}_{\mathrm{L}}$ is the pole at output of the second stage, $\mathrm{C}_{\mathrm{PAR}}$ and $\mathrm{R}_{\mathrm{OA}}$ are the parasitic capacitance seen at the gate of the pass element and the output resistance of the error amplifier, respectively. With carefully selected $C_{L}$ and $R_{\mathrm{ESR}}, \mathrm{Z}_{\mathrm{ESR}}$ can locate between the unity gain frequency and two low frequency poles. As $Z_{\mathrm{ESR}}$ contributes +90 degrees phase shift, this two-pole one-zero system can provide sufficient phase margin. In this way, the system can be guaranteed stable.

However, there are two major problems in the conventional compensation scheme. First, $\mathrm{R}_{\mathrm{ESR}}$ must be some finite value therefore the load transient performance cannot be optimized. Second, the value of $R_{E S R}$ and $C_{L}$ are strictly limited because $D C$ gain and frequency of $\mathrm{P}_{\mathrm{L}}$ of the open loop transfer function will vary with loading conditions. For example, as load current changes from $1 \mathrm{~mA}$ to $100 \mathrm{~mA}$, transconductance of the pass element may be ten times larger, and $R_{L}$ becomes one hundred times smaller. As a result, frequency of $\mathrm{P}_{\mathrm{L}}$ becomes one hundred times larger and the $\mathrm{DC}$ value of the loop gain becomes ten times smaller. In other words, frequency response of the open loop transfer function is different under different loading conditions. So the position of ESR zero is strictly confined in a limited range. That is, $R_{E S R}$ and $C_{L}$ must be designed very carefully.

\section{PROPOSED POLE-ZERO PAIRS CANCELLATION COMPENSATION}

\subsection{Stability of the LDO with Pole-Zero Pairs Cancellation Compensation}

A pole-zero pairs cancellation compensation scheme is proposed to alleviate those conventional design constraints. Fig. 3 illustrates the basic concept of pole-zero pairs cancellation compensation scheme. As a series of pole-zero pairs are generated and arranged appropriately in the open loop transfer function, a sufficient phase margin can be achieved. The frequency of $Z_{\mathrm{i}}(\mathrm{i}=1$ to 4$)$ is designed to be ten times of $P_{i}$. As a result, the phase shift is about 45 degrees over $P_{1}$ to $Z_{4}$ because the phase shift of each pole-zero pair will cancel with each other, and the average slope of loop gain turns out to be $-10 \mathrm{~dB} /$ decade, so a straight line of $-10 \mathrm{~dB} /$ decade can be used to approximate the curve of gain between $\mathrm{P}_{1}$ and $\mathrm{Z}_{4}$. An LDO using this compensation method is stable with different $C_{L}$ and $R_{E S R}$ conditions. The detailed discussions of phase margin and stability can be divided into three cases as below.

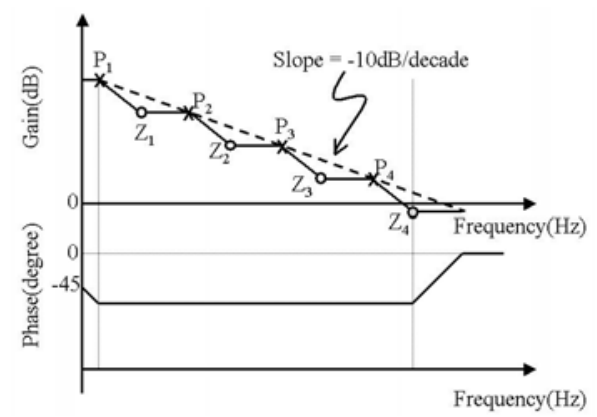

Fig. 3 Frequency response of the pole-zero pairs cancellation compensation

1) Without an off-chip CL: Without an off-chip $\mathrm{C}_{\mathrm{L}}$, $\mathrm{P}_{\mathrm{L}}$ moves to higher frequency than unity gain frequency. As Fig. 4 shows, there are several pole-zero pairs before unity gain frequency, the equivalent slope of gain is $-10 \mathrm{~dB} /$ decade, and $\mathrm{P}_{\mathrm{L}}$ does not affect the phase margin due to its high frequency location. The phase margin is about 135 degrees in this case because pole-zero pairs only provide -45 degrees phase shift.

2) With an off-chip $C_{L}$, without $R_{E S R}$ : Off-chip $C_{\mathrm{L}}$ and $R_{L}$ will generate a low frequency pole $\mathrm{P}_{\mathrm{L}}$. So besides -45 degrees phase shift provided by pole-zero pairs, $\mathrm{P}_{\mathrm{L}}$ contributes at most -90 degrees phase shift. It results at least 45 degrees phase margin and the LDO remains stable.

3) With an off-chip $C_{L}$, with $R_{E S R}$ : When $\mathrm{R}_{\mathrm{ESR}}$ is large enough and that cannot be neglected, it will generate a zero $Z_{\mathrm{ESR}}$ with $\mathrm{C}_{\mathrm{L}}$ lower than the unity gain frequency. As Figure 4 shows, $Z_{\mathrm{ESR}}$ will reduce the slope of gain and increase the phase shift. If $Z_{\mathrm{ESR}}$ is larger than $P_{L}$, that is, $R_{E S R}$ is smaller than $R_{L}$, phase margin of LDO will become larger compared to case 2, so LDO is also stable in this case.

In summary, an LDO with this compensation scheme is stable as long as $R_{E S R}$ is smaller than $R_{L}$, which can be achieved easily. In other words, wherever the position of $\mathrm{P}_{\mathrm{L}}$ is, phase margin of the LDO is at least 45 degrees. 


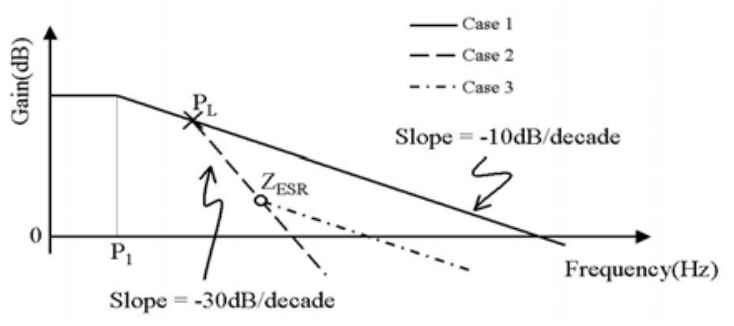

Fig. 4 Frequency response of the loop gain with different load capacitors

\subsection{Pole-Zero Pairs Generation}

The pole-zero pair at the lowest frequency can be generated through a parallel amplifier structure or the amplifier with a feed-forward capacitor [8]. However, several pole-zero pairs are needed for pole-zero pairs compensation. A new pass element structure is used to generate pole-zero pairs with designed positions. Fig. 5 shows the proposed pass element structure, where $C_{1} \sim C_{4}$ can be parasitic capacitors of $M_{1} \sim M_{4}$ or additional on-chip capacitors. If $\mathrm{R}_{(\mathrm{i}+1)}>>\mathrm{R}_{\mathrm{i}}(\mathrm{i}=1 \sim 3)$, the transfer function of $\mathrm{V}_{\mathrm{O}}(\mathrm{s}) / \mathrm{V}_{\mathrm{I}}(\mathrm{s})$ can be written as

$$
\begin{aligned}
& \mathrm{A}_{1} /\left(1+\mathrm{P}_{1}\right)+\mathrm{A}_{2} /\left(1+\mathrm{P}_{2}\right)+\mathrm{A}_{3} /\left(1+\mathrm{P}_{3}\right)+\mathrm{A}_{4} /\left(1+\mathrm{P}_{4}\right) \\
& \mathrm{A}_{\mathrm{i}}=\mathrm{g}_{\mathrm{mi}} \mathrm{R}_{\mathrm{L}}(\mathrm{i}=1 \sim 4), \mathrm{P}_{\mathrm{i}}=\Sigma \mathrm{R}_{\mathrm{i}} \mathrm{C}_{\mathrm{i}}(\mathrm{i}=1 \sim 4) \\
& \text { where } \mathrm{g}_{\mathrm{mi}} \text { is the transconductance of } \mathrm{M}_{\mathrm{i}}(\mathrm{i}=1 \sim 4) .
\end{aligned}
$$

If $g_{m i}<g_{m(i+1)}(i=1 \sim 3)$, the frequency response of $\left|\mathrm{V}_{\mathrm{O}}(\mathrm{s}) / \mathrm{V}_{\mathrm{I}}(\mathrm{s})\right|$ can be approximated by the envelop of the frequency response of the four terms in equation (4), as the solid line in Fig. 6. So this structure generates three pole-zero pairs in the transfer function of loop gain.

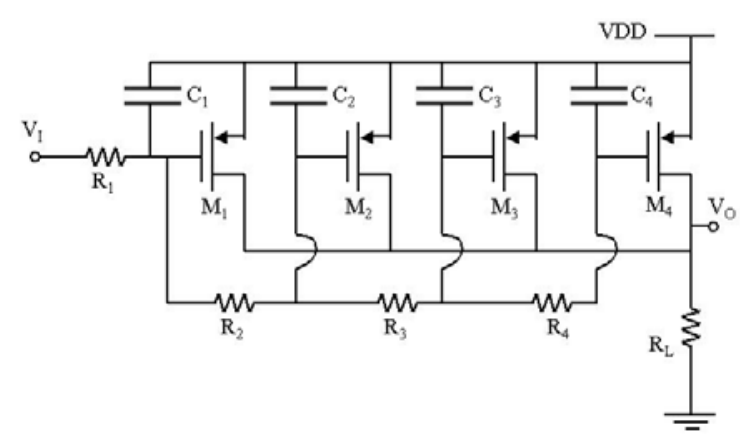

Fig. 5 Proposed pass element structure

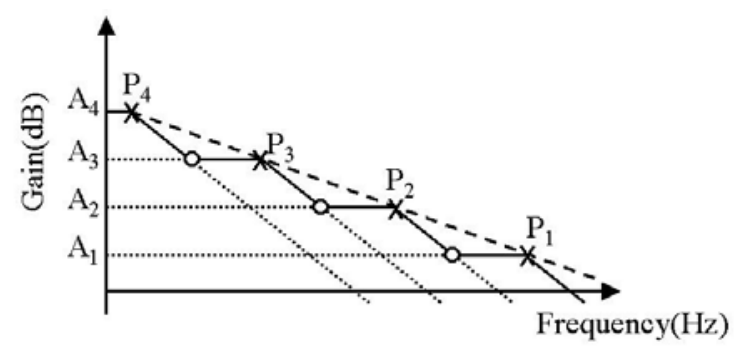

Fig. 6 Frequency response of four terms and asymptote of $\left|\mathrm{V}_{\mathrm{O}}(\mathrm{s}) / \mathrm{V}_{\mathrm{I}}(\mathrm{s})\right|$

\subsection{Circuit Implementation}

The simplified overall schematic of proposed LDO is shown in Fig. 7. It is composed of a differential amplifier with a feed-forward capacitor and a pass element using proposed structure, where $\mathrm{M}_{7}$ and $\mathrm{M}_{8}$ are biased in triode region to behave as resistors. The $\mathrm{V}_{\mathrm{REF}}$ is generated by a bandgap voltage reference. The first amplifier with feed-forward capacitor $C_{C}$ generates the lowest frequency pole-zero pair. Other pole-zero pairs are generated through the pass element. Due to the average slope of gain without $\mathrm{C}_{\mathrm{L}}$ is only $-10 \mathrm{~dB} /$ decade, the DC loop gain cannot be very high because of high frequency parasitic poles. To achieve about $40 \mathrm{~dB}$ DC loop gain for acceptable regulation performance, phase margin for worst case is designed to be a smaller value. The simulated phase margin under different load conditions with different $\mathrm{C}_{\mathrm{L}}$ is shown in Fig. 8 and the phase margin is designed at least 42 degrees.

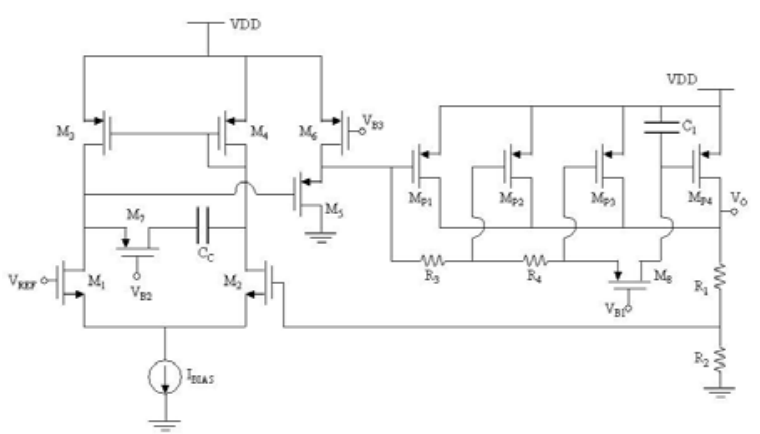

Fig. 7 Simplified overall schematic of the LDO

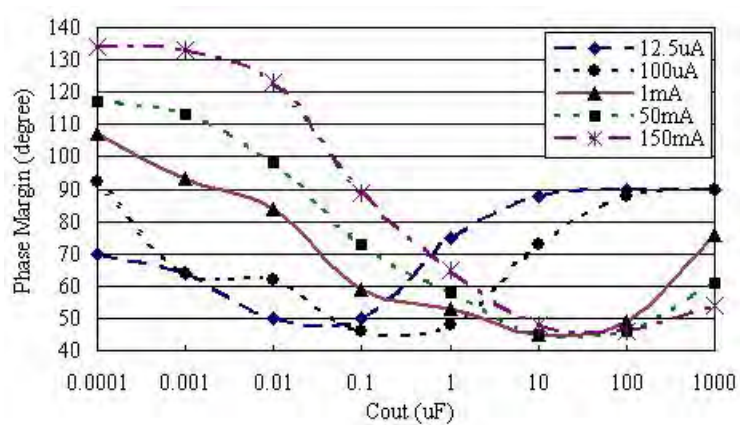

Fig. 8 Simulated phase margin versus $C_{L}$ under different load conditions

\section{EXPERIMENTAL RESULTS}

The proposed LDO has been implemented in $0.5-\mu \mathrm{m}$ 1P2M CMOS technology. It is designed to deliver a maximum load current of $150 \mathrm{~mA}$. The preset output voltage is $2.5 \mathrm{~V}$. Fig. 9 shows the waveforms of output voltage under load current transients using different load capacitors. The load current changes from zero to $150 \mathrm{~mA}$ and back to zero with rise and fall times of 2 us. As Fig. 9 shows, the output voltage is stable with different load capacitors and ESRs. Listed in Table I are the key performance parameters of the LDO. It is noted that $\mathrm{C}_{\mathrm{L}}$ value is not necessarily specified and the ESR stability region 


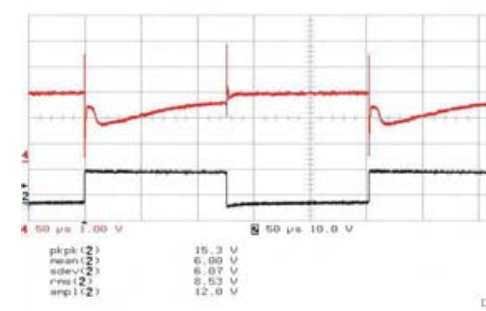

(a)

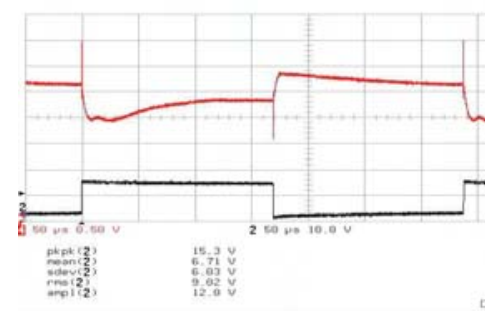

(d)

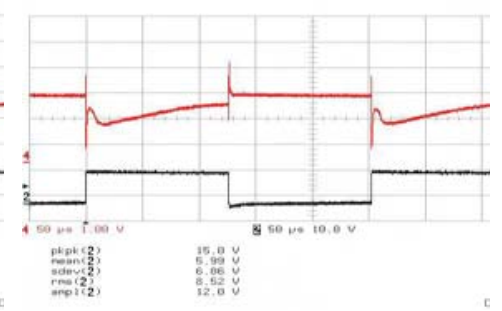

(b)

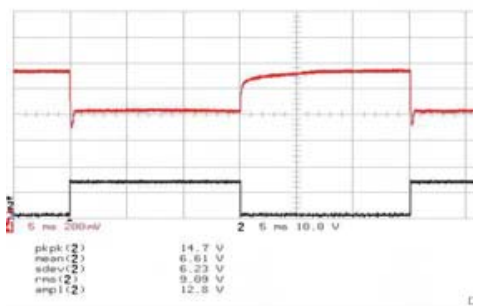

(e)

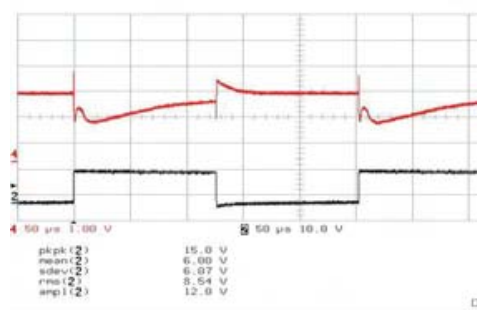

(c)

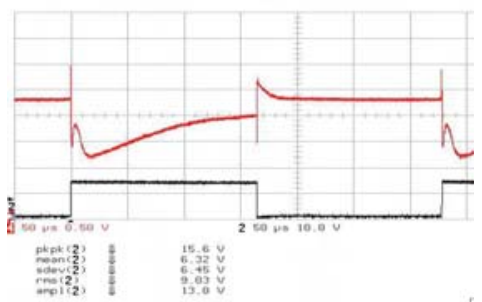

(f)

Fig. 9 Experimental transient response with different load capacitor and ESR:

(a) $\mathrm{C}_{\mathrm{L}}=0, \mathrm{ESR}=0$. (b) $\mathrm{C}_{\mathrm{L}}=10 \mathrm{nF}, \mathrm{ESR}=0$. (c) $\mathrm{C}_{\mathrm{L}}=0.1 \mathrm{uF}, \mathrm{ESR}=0$.

(d) $\mathrm{C}_{\mathrm{L}}=1 \mathrm{uF}, \mathrm{ESR}=0$. (e) $\mathrm{C}_{\mathrm{L}}=47 \mathrm{uF}, \mathrm{ESR}=0.5 \Omega$. (f) $\mathrm{C}_{\mathrm{L}}=0.1 \mathrm{uF}, \mathrm{ESR}=1 \Omega$.

ranges from 0 to $\mathrm{R}_{\mathrm{L}, \mathrm{min}}$. The line and load regulation performance is not so brilliant due to the relatively low DC loop gain (about 40dB in this work).

\section{CONCLUSION}

In this paper, the conventional compensation scheme for LDO and its limitation are analyzed. A new compensation technique using pole-zero pairs cancellation is proposed to improve the sable range for the load capacitor and its ESR value. An LDO with new compensation scheme is fabricated and the load capacitor can range from 0 to any value to provide a stable output voltage. The ESR of load capacitor only needs to be less than the load resistance to ensure stability. The measured transient response is quite consistent with the analysis.

Table 1 Performance parameters

\begin{tabular}{|c|c|}
\hline & Measured Result \\
\hline $\mathrm{V}_{\mathrm{IN}}$ & $3 \mathrm{~V} \sim 7 \mathrm{~V}$ \\
\hline $\mathrm{V}_{\text {OUT }}$ & $2.3 \sim 2.5 \mathrm{~V}$ \\
\hline Quiescent current & $90 \mathrm{uA}$ \\
\hline Dropout voltage & $446 \mathrm{mV}\left(\mathrm{I}_{\mathrm{LOAD}}=150 \mathrm{~mA}\right)$ \\
\hline Load regulation & $1.47 \mathrm{mV} / \mathrm{mA}$ \\
\hline Line regulation & $30 \mathrm{mV} / \mathrm{V}$ \\
\hline $\mathrm{C}_{\mathrm{L}}$ stable range & Not limited \\
\hline ESR stable range & Smaller than $\mathrm{R}_{\mathrm{L}, \min }(16.67 \Omega)$ \\
\hline
\end{tabular}

\section{REFERENCES}

[1] C. Simpson, "Linear Regulators: Theory of Operation and Compensation," Application Note 1148, National Semiconductor, May 2000.

[2] G. A. Rincon-Mora and P. E. Allen, "Optimized Frequency-Shaping Circuit Topologies for LDO's," IEEE Transactions on Circuits and Systems II, vol. 45, no. 6, pp. 703-708, Jun. 1998.

[3] K. N. Leung, P. K. T. Mok, and W. H. Ki, "A Novel Frequency Compensation Technique for Low-Voltage Low-Dropout Regulator," IEEE International Symposium on Circuits and Systems, vol. 5, pp. 102-105, May 1999.

[4] W. Chen, W. Ki and P. K. T. Mok, "Dual-Loop Feedback for Fast Low Dropout Regulators," IEEE Power Electronics Specialists Conference, vol. 3, pp. 1265-1269, Jun. 2001.

[5] C. K. Chava and J. Silva-Martinez, "A Robust Frequency Compensation Scheme for LDO Regulators," IEEE International Symposium on Circuits and Systems, vol. 5, pp. 825-828, May 2002.

[6] K. C. Kwok and P. K. T. Mok, "Pole-Zero Tracking Frequency Compensation for Low Dropout Regulator," IEEE International Symposium on Circuits and Systems, vol. 4, pp. 735-738, May 2002.

[7] K. N. Leung and P. K. T. Mok, "A Capacitor-Free CMOS Low-Dropout Regulator With Damping-Factor-Control Frequency Compensation," IEEE Journal of Solid-State Circuits, vol. 38, no. 10, pp. 1691-1702, Oct. 2003.

[8] G. A. Rincon-Mora, "Current Efficient, Low Voltage, Low Drop-Out Regulators", Ph.D. Thesis, Georgia Institute of Technology, Nov. 1996. 